\title{
What Should a Wildebeest Say? Interactive Nature Films for High School Classrooms
}

\author{
Brian K. Smith \\ MIT Media Laboratory \\ 20 Ames Street \\ Cambridge, MA 02139 \\ bsmith@media.mit.edu
}

\author{
Brian J. Reiser \\ School of Education and Social Policy \\ Northwestern University \\ Evanston, IL 60208 \\ reiserdils.nwu.edu
}

\begin{abstract}
Nature documentaries play an important role in high school biology classrooms, yet they deliver a passive and biased account of the behavior of organisms. To engage students in more active problem solving around behavioral topics, we created an interactive video system called Animal Landlord. Part of a week-long curriculum designed to introduce concepts in behavioral ecology, Animal Landlord presents film clips of the Serengeti lion hunting its prey. Students select and annotate video frames with explanations of their significance to the hunt, compare annotations across films, and ultimately generalize a qualitative model of predation behaviors. This paper discusses the motivations for changing the nature of documentary use in the classroom, the ways in which we change the form of traditional narration for pedagogical purposes, and the interactivity that emerges in the social context of the classroom.
\end{abstract}

\section{INTRODUCTION}

New reform efforts in education (e.g., [17]) attempt to move students away from passive textbook and lecture activities by advocating more student-directed activities. Providing students with rich problem settings in which they can engage in iterative hypothesis generation and testing and explanation of causal relationships may result in more productive learning that shallow exposure to a broad base of content [4, 23]. Many computerbased learning environments have been developed to provide interactive settings for students to engage in realistic activities.

However, many educational multimedia systems do not conform to these reform standards. We still see systems that essentially integrate traditional educational media into an "electronic textbook" [29]. These systems provide opportunities to explore content nonlinearly, but they still place the student in the passive role of media consumers. As well, many of these systems attempt to stand alone, encouraging a one-on-one interaction between computer and student [19]. While there is nothing wrong with personalized instruction, social collaborations with peers and teachers are crucial to learning, and this is often neglected in the design of educational multimedia.

In ACM Multimedia 97 Proceedings (pp. 193-201). New York: ACM Press.
Multimedia designers organize and relate content materials to make particular kinds of experiences accessible to students. In some sense, many of the interesting learning opportunities occur during this process, as designers are forced to interpret meaning from media and to develop coherent storylines. Perhaps if we want students to assume more active roles in their learning, we should let them engage in this process. Instead of imposing meaning on a database of media, we should allow students to do the interpretation and plot development. More so, we should allow them to collaborate with teachers and other students in this process to encourage multiple viewpoints on the construction of meaning. The goal of our research is to empower students to become media producers.

As part of the BGuILE (Biology Guided Inquiry Learning Environments) project [30], we have constructed an interactive multimedia tool called Animal Landlord. Designed for high school biology courses, Animal Landlord engages students in concepts from behavioral ecology, such as resource competition, social organization, and optimal foraging theory. We provide a set of computational tools to support students in creating "narratives" of a corpus of video clips. The video allows students to observe the behaviors of lions during their hunts. Use of the computer environment is meant to help students collect "field data" which are then used to generalize larger, explanatory structures accounting for the causal influences on lion predation and prey evasion.

Traditional nature films, where visual imagery provides firsthand accounts of various flora and fauna, can be useful observational tools. But the film itself is simply a starting point for further discussions and integration with other curricular materials. Without complementary activities, students may simply view the film as a novel relief from their textbooks. Instead of compiling video materials into a multimedia presentation system, we have students essentially create their own documentaries using video as a primary data source. Educational film is largely a passive medium, and our goal is to make it less so by using it as a foundation for more active observation.

Our goal is not to provide hypermedia systems for browsing information about animal behavior, but to create a tool for students to observe and construct explanations of complex behaviors found in video. But a tool is not enough, for students also require assistance in understanding how to construct explanations from video. Because they are so used to passive viewing of documentaries, we have had to iteratively design and refine structures that help them explain visual events in meaningful ways. Some of these structures exist offline, as part of the classroom culture. As there are established norms of interactions in 
classrooms, we have had to introduce interactive video within existing social constraints.

This paper focuses on two issues that we have faced in reconstructing the nature documentary into an interactive, learning experience. First, we look at the use of documentary films in classrooms to understand how interactive video can change traditional patterns of activity. How can multimedia be used to change the typically passive event of watching a film into the active, social development of scientific ideas? Second, we consider some preliminary results related to learning outcomes. If we change the use of film in classrooms, can we also see changes in the ways that students reason about content issues?

\section{FROM NATURE FILMS ...}

Film appears to have benefits as an educational medium [4, 21, 32]. Visual events are rich with opportunities for students to pose their own questions. Teachers can encourage students to think about interesting events or anomalies present in the film, including issues not explicitly mentioned in the narration. The ability to view dynamic events can also facilitate understanding - watching a lion chase its prey is much different than simply reading about its speed. As a result, video establishes a context for applying domain skills and concepts, for seeing how scientific abstractions manifest themselves in the world.

Yet, many educators have attacked educational films for being little more than "illustrated radio shows", providing compelling narratives but few opportunities for students to reflect on the content [11]. Film narrations may suffice for topical overviews, but they rarely present in-depth explanations of how and why animals behave as they do. In this sense, the narration is similar to many high school textbooks, presenting factual, surface accounts of the organism while excluding much of the interesting science $[19,25]$.

Nonetheless, there is evidence that viewing science films results in knowledge gains [9, 27]. However, it has also been shown that watching a nature film results in the same learning gains as simply listening to the film's narration without the video [9, 32]. In cases where we have presented documentary footage without narration in high school classrooms, students typically complain about the lack of a textual guide - "How are we supposed to know what is happening?" Narrative focuses attention on relevant visual events, acting as the primary source of knowledge in documentary films $[8,32]$, yet there is a great deal of implicit information in the video that students can also observe and explain for themselves. Unfortunately, the inclination to passively listen to the narrator impedes such student-directed learning.

Another interesting result is the relationship between student misconceptions and biological films. Silverstein and Tamir report that students often generate fewer causal explanations of topics in a film after viewing [27]. These causal explanations found in pretests were replaced by anthropomorphic (relating animal behavior to human behavior) and/or teleological (relating behavior or structural features to a goal rather than a mechanism) explanations. On the other hand, students were able to regurgitate information transmitted through the narration. This implies a difference between factual knowledge and reasoning about biological phenomena. Students are adept at absorbing the former, while the latter requires additional knowledge excluded from the narration and/or implicit in the visual scene.
There are a number of issues facing the use of educational films more actively in science classrooms. For one, the presented material is primarily factual, neglecting interesting domain processes in favor of straightforward outcomes. They also tend to ignore the numerous interactions between an organism and its environment that lead to its behaviors. Secondly, the video itself does not appear to be the major contributor to student learning; the narration is the dominant purveyor of information. These two areas may be related, as conveying outcomes may be more congruent with the aesthetic concerns of film makers than discussions of processes. Finally, these films present a single, linear story about the visual events at the cost of limiting potential problem solving around the video itself. This discourages students from making their own observations and interpretations, again leading to the passive reception of information. While all of this may suffice for casual viewing, we would like to see students reflecting on and explaining the rich, visual data found in nature films.

It is worth noting that similar arguments have been raised against educational multimedia. Many early systems merged multiple media in interesting ways, yet they still focused on delivering presentations to students $[19,29]$. Nonlinear browsing of hyperlinked materials may alleviate some of the passivity associated with film, but the software designer is still impinging a storyline on the user that can also be viewed passively [6].

\section{TO INTERACTIVE NATURE FILMS ...}

Our goal is to change the ways that documentaries are used in classrooms by shifting students from recipients of content to producers of media artifacts. Textbooks and traditional school curricula bias students towards views of science as factual information that is to be accepted without argument. Documentary films often do the same, presenting carefully crafted stories suggesting a "right" way to view a complex phenomenon. In a sense, students perceive the development of scientific knowledge as data simply waiting to be discovered. We would like to help them understand that it is experimentation, argumentation, and iterative refinement of ideas that lead to the "truths" found in science. Instead of simply understand facts, we would like them to understand the reasoning strategies that underlie scientific endeavors.

New science reforms calling for active problem-solving argue that learning is an continual process of engagement with ideas and materials [17]. In typical classrooms, student performance is only assessed by the teacher and rarely shared with other students. More and more, there is an shift away from this, calling for students to make their understandings explicit as observable artifacts which can be examined and shared with teachers and peers. This process of sharing and creating work with others can lead to classroom cultures in which students come to critically interpret and learn from the work of their colleagues.

What does this mean for the development of educational multimedia? We want students to begin looking closely at visual information, relying less on the narrator and more on their own observations and interpretations. In some sense, we want them to become multimedia researchers, decomposing video into events, analyzing and drawing connections between these events, and collaborating with others to construct meaningful representations of the visual data [12]. Instead of creating the digital analog of the nature film, we are trying to create envi- 
ronments for students to learn by making their own interpretations of visual information and assembling their own narrative accounts.

The development of such tools can be seen in projects like MultimediaWorks [18] and MAD [2], authoring environments used to create multimedia stories and movies. Goldman-Segall's Learning Constellations is a video annotation tool used by adults and students to create ethnographic accounts of their school culture [12]. There are also several tools used by students to analyze properties of motion from digital video [7, 20,21]. With these, students take measurements of physical phenomena directly from video clips to tell quantitative stories about distance, rate, and time relationships. Our work is similar to these systems but differs in its focus on analysis of complex behavior. Our goal is for students to describe causal interactions and to analyze variation across multiple video episodes to generalize qualitative models of behavior.

There is also a need to acknowledge the role of social interactions in learning. Many multimedia technologies have been designed for singular use, acting as environments for individual students to peruse information and practice skills. For some domain areas, this is a useful tactic; formalized, algorithmic practices, for instance, may be better taught through self-paced, individualized instructional methods [1]. But many high-level reasoning skills, such as hypothesis generation and testing, may be better facilitated through collaboration with others who can pose opposing viewpoints. Instead of designing multimedia as a more efficient purveyor of knowledge, we should be designing learning interactions, of which the computer is a central tool [19].

Nardi et al. depart from the use of video as "talking heads" in a system that facilitates collaboration during neurosurgery [16]. Video acts as a prop for coordinating activity in the operating room, but it also plays a learning role for students, as it can later be viewed to understand the real-time demands of expert practitioners. Collaboration around video also plays a role in Media Fusion [3], a system that integrates QuickTime video with various quantitative data tools (e.g., spreadsheets) for students to create narratives around video. The goal of these projects is to integrate video media seamlessly into the norms of social interaction. Our work is similar in this regard; the interactions take place in the classroom, and video is used to construct causal analyses of behavior.

In our work, students create explanatory narrations for a corpus of video clips. To do this, they view documentary footage seeking answers to an assigned question (e.g., "Why is the lion a 'bad' hunter?'). Instead of simply watching films, students edit and manipulate video frames as a primary data source. Gradually, they move from raw video footage to working with evidence in the form of significant frames. These frames are used to construct more complex generalizations of the processes under observation. As we will describe, this means moving away from linear, text narratives to a format that allows interactions and causal connections to become more explicit.

Student narrations are concrete products shared in small-group and whole-class interactions. In these group sessions, students shift from creators to critics, arguing over the validity of each other's work and working together to develop better interpretations of the film. In some sense, the exercise is a form of "interactive documentary", for students manipulate video frames to generate explanations of behavior, creating a narrative framework. It is not interactive in the sense of nonlinear, multimedia presentations authored or viewed by the user (e.g., [15]).

It is also interactive in the sense described by Brooks when he points out that the most interactive of movies may be The Rocky Horror Picture Show [5]. While researchers continue to develop complex technologies for computer story presentation, Rocky Horror fanatics are content to dress, act, and sing like their favorite characters ... all in the middle of a crowded movie theater. Brooks' observation is that interactive experiences do not necessarily begin with the modification or nonlinear browsing of films; audience participation can alter a film's experiential qualities.

Our students are the audience, and the classroom is the movie theater that will house the interaction. We have an additional player in the environment, the teacher, and we will discuss teacher roles in sustaining the interactive nature film in a later section. The activities we will describe digress from the linear viewing of the nature documentary, changing passive viewing into a more active experience. Interactivity emerges as students and teachers collaborate to solve problems posed by the video, not as a result of altering the film sequence.

We are trying to overcome the conceptions of science and nature film as static facts by having students construct qualitative models of visual events. Instead of simply watching digitized films that explain science as truth, students will construct their own interpretations using video as data, interpretations that parallel those found in the ecological literature. The process of interpreting and explaining visual data is largely social, with students collaborating to create what would normally be called a narration. From this, we hope that students will gain a better understanding of the scientific endeavor and better strategies for reasoning about complex, behavioral phenomena.

\section{ANIMAL LANDLORD}

Forty-four lion prides within a $2,000 \mathrm{~km}^{2}$ area of Tanzania's Serengeti National Park have been under continuous scientific observation since 1966 [28], and our curriculum focuses on their hunting behaviors. When we ask students how often they think lions successfully capture their prey, their predictions are quite high $(50-90 \%)$. In reality, only $15-30 \%$ of all attempted hunts actually result in a successful capture [22]. This mismatch between the lion's mythology and scientific observations raises curiosity and sets the stage for learning topics in behavioral ecology such as social organization, resource competition, variation between individuals and species, and environmental pressures.

To understand why lion hunting success is lower than expected, one needs to understand the causal interactions between the lion, its prey, and the environment. Observation of the creatures would likely provide some insight into these interactions, but sending high school students to the Serengeti plains is rarely an option. Instead, we use video to achieve the same end. We have found that students are quite good at watching lion films and generating flowery tales of predator versus prey, but their spontaneous narratives contain few causal analyses or mentions of dependent variables. In a sense, they are adept at creating narrations that one might find in a traditional documentary, but they are not naturally making detailed observations and interpretations of the visual events. With this in mind, we created an 
interactive video system to explore the behaviors of the Serengeti lion.

\section{Documentary Story Structure}

Our pedagogical focus is on the acquisition of both content and strategic knowledge. By content, we do not mean that students should become experts on lions. Predation behaviors serve as an example from which discussions of resource competition, social cooperation, and evolutionary adaptations can occur. Animal behavior is typically relegated to a single textbook chapter, mostly presenting terms for memorization with a few examples. We hope to provide deeper understanding by providing a rich example and then later applying terminology to features of it.

As for strategic knowledge, we would like students to gain an appreciation for the subtleties of scientific observation and explanation. Although lab assignments are common in high school biology, they tend to have a "cookbook" flavor to them, and students rarely have opportunities to setup experimental conditions and to explain results for themselves [23].

One of the steps in our iterative design was to look at 200 narrative descriptions of lion hunting videos written by high school students before they used Animal Landlord. These paragraphs tend to obscure the salient events in the hunt, and they also tend to carry additional embellishments and inferences not present in the visual scene. A typical example appears below:

They sort of stalked their way along and were cautious not to make any sudden movements until the end. The lion quickly charged a (sic) unsuspecting zebra which didn't have time to think or run and this made the lion successful in catching his prey.

We try to help students shift from these narrations to causal explanations of behavior. This requires explicit focus on the relationships between events, a focus on the components of a "good" explanation. Not only should students understand how these components come together to form a scientific argument, they should also understand how to detect them during their observations.

Ecologists have expertise which helps them focus their observations and explanations when doing field observations. Through conversations with ecologists and a study of the literature, we developed an investigation model [30] which attempts to capture important features of ecological experimentation and argumentation. High school students lack this expertise, and we must assist them in acquiring these practices.

The investigation model can be summarized as:

- Decompose behavioral observations into related, constituent actions.

- Compare across similar events, looking for variations.

- Identify factors accounting for these variations.

- Relate factors back to behaviors to form an evolutionary explanation.

Animal Landlord was designed around this investigation model. A software annotation tool focuses students on the first point, detecting intermediate actions that lead to an outcome. Comparison and identifying strategic factors that vary across events is also facilitated through a visual comparison tool. A modeling activity at the end helps to link everything together, forming a qualitative explanation of hunting behavior.

Text narratives tend to cloud the details of intermediate events that have a causal role in the hunt outcome, while the investigation model leads to a story structure highlighting these dependencies. The model also assists the development of a final story by suggesting relevant areas to focus on during observation and explanation. The software eases the process of analysis by offering tools based on the model.

\section{Annotation}

Animal Landlord provides nine video clips, each depicting a different way in which lions obtain their food. The films vary across factors such as size and composition of the lion/prey groups, prey species, time of day, hunting methods (stalking, ambushing), and hunt success or failure. This allows students to make comparisons between films to identify strategic factors influencing hunt outcomes and their range of possible values. Groups of three to four students work with these clips, presented as QuickTime video without narration on the computer, and use a tool which allows them to capture and annotate frames of the film to explain their significance (Figure 1). Ultimately, we want students to produce explanations around the film clips, and their initial annotations will serve as the basis for these.

The menu in the center of Figure 1 shows a number of possible interactions between predator and prey. This feature was added after initial tests with students, where we noticed that students did not spontaneously decompose the films into such actions. Instead, they would grab a single frame, generally the final one, and make comments such as, "The lion failed its hunt because it was slower than the warthog." This suggests that hunting is simply viewed by many as an outcome - the predator eats or goes hungry. It is important that students see the causal steps leading to an outcome, and the addition of this menu greatly increased the quality of annotations in later sessions. Not only were students able to identify the features present in the menu, but they also identified additional features (which can be added to the menu and shared by other groups), possibly as a result of spotting unusual events that were not on the action menu.

A large number of our students would write annotations such as, "The predators changed their mind and let the prey get away because they are in a playful mood." These comments conflate observations of the film with possible inferences or conclusions. In a scientific analysis of behavior, such distinctions are critical, so we created two types of annotations. In the "Observations" column, students comment on the actions that lead to the selected behavior label. This information leads to the second column, "Interpretations/Questions", where students make inferences about the reasons for a particular behavior and can note questions that they might have about the visual events.

\section{Comparison}

Every film tells a particular story about a hunting encounter, a story that has been carved into relevant events by the students. The next task is to understand how these stories can be assembled to create a complete picture of lion predation. That is, we can view predation as a space of activities ultimately leading to one of two outcomes - either the prey is captured or it escapes [14]. Along the path to these outcomes are a number of "decision points" that are influential to the predator's success or failure. Mapping this space is useful for understanding the interactions 
between the predator, its conspecifics, its prey, and the environment.
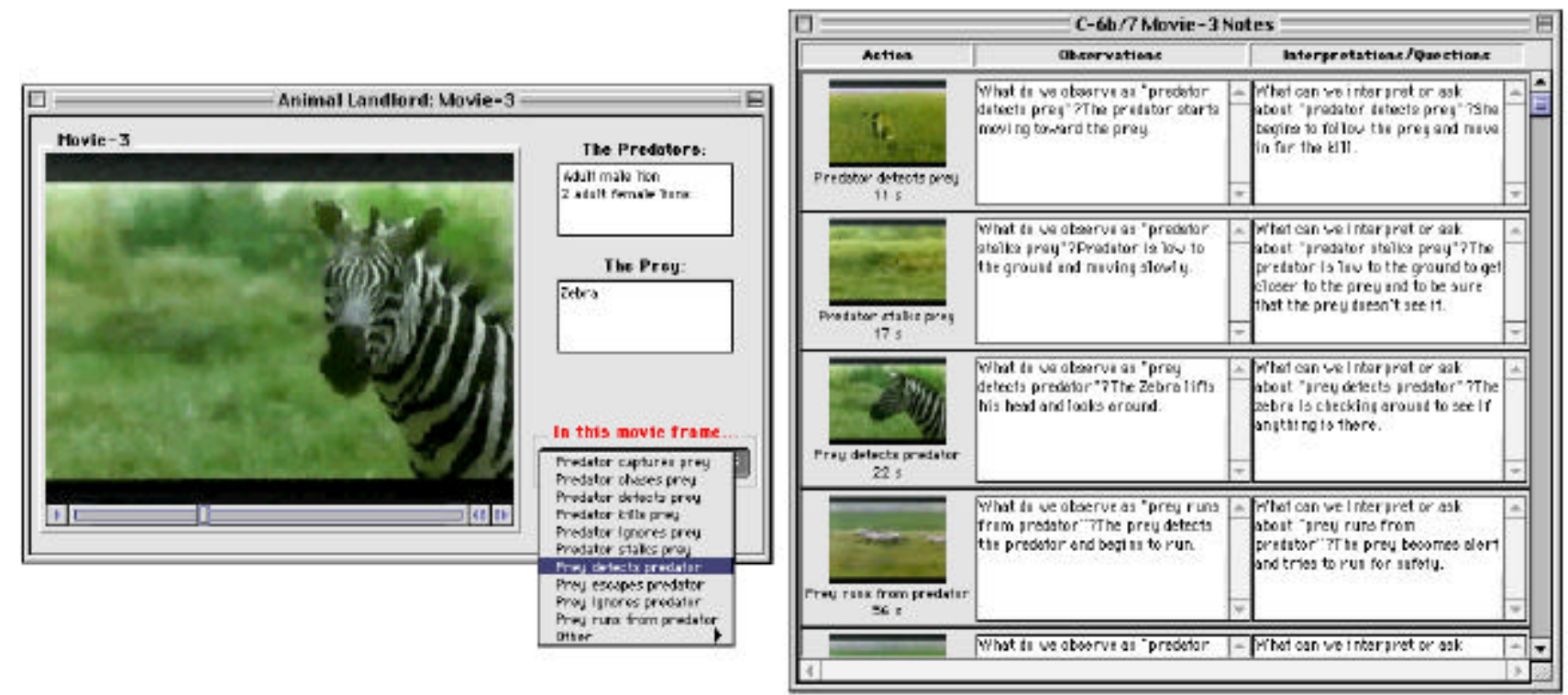

Figure 1: The Animal Landlord's movie viewer and annotation tool. The exposed menu is used to label movie frames from a palette of actions. The frame is sent to the annotation window on the left where students make observations and interpretations around the event. Alternatively, students can directly drag and drop a frame from the movie viewer to the annotation window.

The first step in building a decision tree of the hunt space is to examine variations across films. We provide a "light table" for students to examine the results of their annotations (Figure 2). Students engage in a conversation around their initial annotations once loaded into the light table. They can select particular actions to see where similar events occur across films (Figure 2 shows the light table aligned at "Prey runs from predator"). Lining up similar actions in the light table often reveals differences in the surrounding states that may be important to understanding the hunt space. For instance, in some cases, a predator might not stalk before chasing; this may suggest something about the necessity of performing the action.

\section{Decision Trees and Strategic Factors}

The light table provides facilities for explicitly comparing films, and students use it to understand variations across hunts. From the table, they construct decision trees that represent the space of hunting outcomes. Currently, this is done offline, on large sheets of butcher block paper - Figure 3 shows an example decision tree created from three of the films. Essentially, students create a qualitative model of predator-prey interactions, specifically looking at decisions made during predation. These models are similar to those found in the ecological literature [14].

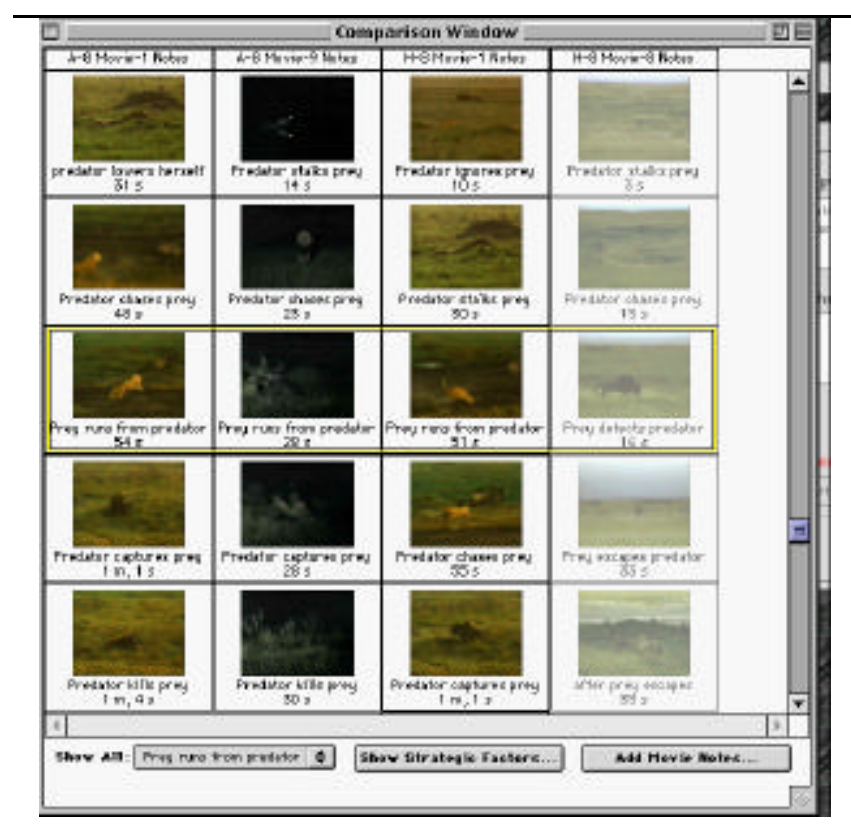

Figure 2: Animal Landlord's comparison light table. Students can align actions that occur across films. This table is aligned on "Prey runs from predator", and all films containing the action are 
highlighted in yellow. The film on the right does not contain the action, hence its column is grayed out.

Decision trees are useful for tracking the potential progress of a hunt, but it is also useful to consider the strategic factors associated with each node in the tree. For instance, a successful stalk relies on a number of factors, such as the amount of cover available, the sensory devices of the target prey, and so on. As students construct their decision trees, they also identify selection pressures influencing the shape of the hunt space. Pressures can either be added to the paper decision trees or linked to actions in the light table using an editing tool in Animal Landlord.

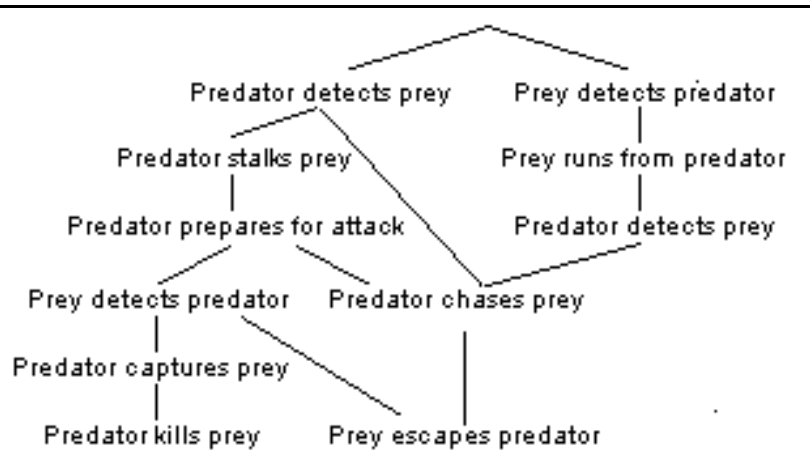

Figure 3: A partial decision tree generalized from three films. Students create these trees on large sheets of butcher block paper to model predator-prey interactions during hunting encounters.

All of this takes place over a week and concludes with discussions of the trees and strategic factors discovered by students. Potential evolutionary reasons for the structure of the tree are brainstormed (e.g., Why do the female lions do most of the hunting?). Teachers will also select nodes in the trees to talk about optimal foraging theory and energy (e.g., Why do the predators decide to give up the chase?). The trees and strategic factors are also used as "predictive" models. Students watch additional films, try to fit them into their models, and revise their structures where necessary.

\section{Animal Landlord's Behavior}

Animal Landlord runs on Macintosh compatible computers and is implemented in C++ on top of Apple Computer's QuickTime Media Architecture. Although we have only dealt with clips of lion predation in classrooms, the tool was designed to handle arbitrary visual content, and using standard resource editors, it is relatively straightforward to change the video clips and action selection menus. People have expressed interest in using the toolset for domains such as golf instruction, social studies, English literature, and ethnographic research, and we are interested in pursuing some of these to see what additional tools might be needed to make sense of visual data in those areas.

The benefits of the software tools seem to be their reflection of the investigation model described earlier. Students should realize that there can be multiple, competing explanations for an organism's behaviors, and structures like the annotations and the decision trees help to emphasize differences in student work. The light table proved to be a valuable asset during student col- laboration, for they could easily inspect intermediate actions and detect salient variations. By scanning films in the light table, strategic factors could also be derived from the films.

After a week of activity, students produce film annotations and decision trees, but they never create text narrations similar to those in nature films. We are about to implement a module that will allow students to sequence the original video clips, add audio tracks, and actually produce a documentary film. We suspect that moving the information "out of the box" so that it can be shared outside the classroom would be a powerful motivator for students.

\section{CLASSROOM THEATRICS}

Thus far, we have had three separate outings in Chicago-area high schools. Each session was conducted in four classrooms, for a total of 12 classrooms and $300+$ students. In each iteration, we were looking to see what kinds of activities occurred during the curriculum. In particular, we were concerned with the types of conversations taking place, the role of the teacher in developing these conversations, and the work produced by students. We were also trying to identify components of the design that could be improved to help students learn from the activity. In this section, we discuss some aspects of Animal Landlord's design that have emerged from these classroom trials as well as some preliminary learning results.

\section{The Teacher}

Each session has made it evident that teachers orchestrate classroom interactions, and their role in the execution of activities is paramount to the success of computer-based learning environments. We are only beginning to understand and characterize teacher influences on the computer implementation, but some preliminary observations are worth reporting.

First, we realized the importance of discussions before, during, and after work with Animal Landlord. The computer does not provide "content" in the manner of textbooks, nature films, and, for that matter, many multimedia programs. As the activity progresses, teachers talk with students in small-group or wholeclass discussions, directing their activities and encouraging argumentation around their findings. These discussions tend to be student-centered; the teacher's primary role is to respond to their queries and to suggest directions for investigation. Students do their own share of independent discussion as they argue around the films to construct their annotations and decision trees, and these arguments spill over into classroom discussions. Ultimately, learning seems to emerge from student-initiated discussions fueled by the observations made on the computer. This is very different than traditional lab activities in classrooms where discussions are driven by teachers.

Teachers are also important in setting up the task, for students are not accustomed to annotating films. We provided filmed materials on videocassette for whole-class discussions prior to computer work. In our first classroom deployment, teachers talked through these videos to provide examples of decomposing hunts. However, this was not enough preparation for the annotation task, as we found that many students would simply mark the final frame of the film. Others would try to find a frame for every item on the action menu, leading to annotations that had the prey simultaneously escaping and being killed.

Our next trials included this same videotape, but we added footage of chimpanzees hunting red colobus monkeys. This film 
is the only one in our curricular unit that has an audio narration. Teachers lead students through an in-depth analysis of the hunt events, and they also point out pros and cons of the narration. For instance, this particular film does an excellent job of explaining the social interactions of the chimpanzees. On the other hand, it mentions that chimps hunt in the wet season without explaining why that might be. Teachers pull out these subtle distinctions in the quality of explanations and use them to model what they expect of students during computer work.

The resulting annotations are much different than the previous outing, with students accounting for more events in each film and adding more of their own labels for actions. This suggests the importance of this type of task modeling, and therefore, we have used the chimp film as an anchor for the complete curriculum. Teachers constantly refer to it to help students understand how to create their decision trees, how to think about strategic factors, and so on.

\section{The Classroom}

Many have asked us why students create their decision trees on paper rather than on the computer. In a sense, we are exploiting the existing culture of the classroom. Students and teachers are accustomed to presentations around large paper artifacts, and we see no need to violate this convention; indeed, we suspect that doing so would drastically change the quality of class interactions. While we could easily develop a tool for sketching decision trees, the activity around a large sheet of paper is profoundly different than what one would find at a computer. It is common to find creative additions to these trees as students flex their aesthetic sensibilities. Paper can also be tacked to the walls, allowing whole-class discussions to take place around central artifacts.

Another interesting factor involves the location of computers. In one trial, we alternated days between a computer lab for video activities and the classroom for discussions. In these classes, students focused on completing computer tasks and only occasionally reflected on the events in the film. Class discussions suffered because there was no way for them to refer to films they seen the day before. It was as if students could not (or were unwilling) to connect activities performed in drastically different locations.

For our remaining trials, we brought machines into the classroom. In these, students spent a great deal of time interacting around the video. Even after an assignment was completed, they would continue to use the video as evidence for their arguments. Because the machines were always available, teachers could use them as conversational props during class discussions. Continuity between class activities and the interactive video environment was maintained. We have yet to do a formal analysis to see whether there are measurable learning differences between these two situations, but student behaviors were clearly different.

There are lessons to be learned from these observations of teachers and classrooms. Multimedia systems are often developed with a focus on the enabling technology and not the interactions that take place around it. While there have been studies of deployments of computers to classrooms (e.g., [13, 25, 26]), we do not have a good understanding of the relationship between teacher practices and the success of interactive computing in schools. Our next efforts in classrooms will be to understand more about student/teacher interactions throughout the academic year and how these influence their adoption of these learning activities.

\section{The Students}

It is obvious from classroom observations that fourteen year old students are motivated to watch videos of large creatures chasing down and eating larger creatures. Although there were some initial concerns about gender differences around the subject of predation, we find that both girls and boys are engaged in the activity. Only one high school girl has ever said to us, "You know, this is entirely too violent for high school students."

This "violence" is the center of the conversation as students become acquainted with the video corpus. As time goes on, discussions become more sophisticated and centered around the video annotation process. For instance, herbivore vigilance is well studied in behavioral ecology (for example, [24]), yet we have never found mention of the topic in high school textbooks. Nonetheless, it occasionally emerged in our classrooms as students compared films using the light table. As they noticed patterns of animals alternating between feeding and scanning the area, they began to generate theories about how often animals need to scan, differences in scan times for different animals, and so on.

Students also became aware of their use of terminology as they looked for selective pressures. Initially, many of them would say things like, "Well, the lion is just being sneaky." Eventually, they began to question one another about what was a decision factor and what was a byproduct of the factor. With the help of the teachers, students began to think more about what features contributed to "sneakiness", taking a more global look at the visual data. Sneakiness would become a product of multiple factors, such as amount of cover in the area, prey type, time of day, and so on. Again, these kind of discussions present important opportunities for learning.

We are only beginning to formally analyze the annotations, but it seems that many students are still having difficulties articulating distinctions between observations and inferences. Unfortunately, the current design does little to help them, as the annotation products are generally neglected after their creation. When comparing films, students focus on the plot structure of their annotated films, but they rarely compare their justifications for each event, despite being able to do so from the light table. Our teachers suggested that we find ways to better integrate these explanations into the activity, and we are hoping that the addition of facilities to actually narrate the video from their annotations will be a step towards this.

We are in the process of reviewing and analyzing data for learning outcomes. Collected data include interviews with students, video observations of classroom interactions, all products created by students, and pre- and posttests. These pre- and posttests asked university level, essay questions about various behavioral issues, as well as testing students' observational skills around video. The data reported here represent pre-post results for two classrooms ( $n=44$ students)

Consider this essay question that appeared on the pre- and posttests: What limits the amount of prey consumed by a predator? Initially, students had responses such as, "If they're not hungry, they won't eat," and, "They know they have to save 
food for times when prey are scarce." After going through the Animal Landlord curriculum, we would hope to see more elaborated responses incorporating relevant biological conceptions. That is, we expect to see explanations tied closely to behavioral phenomena, and we would also expect to see more causal explanations as a result of decomposing, analyzing, and linking related visual events.

For each essay question, we are looking at the number of points raised (e.g., "size of prey is important"), and the number of justifications that back these points (e.g., "size of prey is important because large animals may be difficult for a predator to subdue"). These numbers give us a rough idea about what students could articulate before and after the intervention.

The number of points and the number of justifications increase significantly from pretest to posttest (Table 1). The number of points raised for each question increase, $F(1,42)=28.63$, p < .001 , as do the number of justifications for each point, $F(1,42)=$ $14.14, p<.001$. There were no significant main effects between teachers in these areas.

We also looked at student justifications to see if students were grasping the content of the exercise. The justifications were coded for the presence of behavioral ("If predators are in groups, they must share their kill"), environmental ("The time of day affects the predator's ability to capture prey."), and energy issues ("A predator might not chase if the prey cannot provide enough energy."). We also looked to see how often students made explicit reference to interactions between predator and prey ("The time of day might benefit the predator if the prey cannot see in the dark.") since these relationships are stressed in the decision tree activity. There were significant increases for all categories, and, again, there were no differences between classrooms (Table 1).

\begin{tabular}{|c|c|c|l|}
\hline Measure & Pretest $\bar{X}$ & Posttest $\bar{X}$ & Comparison \\
\hline \# Points & 2.43 & 3.93 & $\mathrm{p}<.001$ \\
\hline \# Justifications & 1.25 & 2.41 & $\mathrm{p}<.001$ \\
\hline Behavioral & 0.75 & 2.32 & $\mathrm{p}<.001$ \\
\hline Environmental & 0.11 & 0.36 & $\mathrm{p}<.05$ \\
\hline Energy & 0.02 & 0.41 & $\mathrm{p}<.001$ \\
\hline Agent interaction & 0.02 & 1.14 & $\mathrm{p}<.001$ \\
\hline
\end{tabular}

Table 1: Six measures coded for in student pre- and post tests. The mean values are combined across the two classrooms. Comparison values are based on ANOVA calculations ( $F$ (1, 42)).

We also found a significant interaction effect of teacher with two of these learning measures - the number of points raised per essay, $F(1,42)=5.67, p<.05$, and the number of environmentally related explanations, $F(1,42)=4.38, p<.05$. This suggests a difference in teaching styles that can be explained through classroom observations. Students of one teacher had larger gains in both areas, and this teacher also spent more time up front with the introductory videos, walking students through the observational process.

Reasoning about behavior often results in misconceptions on the part of students. These misconceptions tend to manifest as anthropomorphic and/or teleological explanations of behavior $[10,31]$. Earlier we mentioned a study where these types of misconceptions increased after the viewing of documentary films [27]. For this reason, we wanted to assess whether students using Animal Landlord had similar changes in reasoning.

Our data show different trends than those reported by Silverstein and Tamir [27]. We coded for anthropomorphic, teleological, and causal justifications, and a combined chi-square analysis for both classrooms shows significant decreases in anthropomorphic explanations and increases in teleological and causal explanations $\left(\chi^{2}(2)=14.97, \mathrm{p}<.001\right)$. It appears that some students initially holding anthropomorphic rationales for behavior are shifting towards teleological conceptions, thus accounting for the increase in that category. The increase in causal explanations is also promising, as it shows students learning to articulate scientifically plausible explanations.

The data suggest that students are learning during their interaction with Animal Landlord. More concepts are being articulated and justified by students, and the justifications are all related to issues in behavioral ecology. More important, these justifications become more causal, suggesting that students are learning to reason scientifically about behavioral phenomena. However, there are a number of unanswered questions that remain to be tested in future work.

Because we lacked a control group, it is unclear whether Animal Landlord results in learning gains beyond more traditional methods of instruction. The material covered in our classrooms far exceeds that found in a high school biology textbook, but we do not have the data to show that Animal Landlord students have a better grasp of the material than students in traditional classrooms. We also cannot say anything about active versus passive learning with respect to film. It is clear that our students are conversing about more sophisticated issues than those found in nature films. Yet, if a nature film were to discuss these topics, would students learn just as much? Or is there something about actively interpreting the video that contributes to the development of strategic reasoning. In future, we want to study these two issues in more depth.

\section{CONCLUSION}

This is but one approach to deploying interactive video to classrooms. We have presented an overview of our research, highlighting the use of film in classrooms and how we have tried to change this activity with multimedia tools. Most important for us is the notion of interactivity developed in the paper. Instead of creating a relationship between a single user and a machine, we have tried to cultivate an atmosphere where an interactive video system is the centerpiece for emergent collaboration, problem solving, and explanation. Understanding the dynamics of this relationship require further study, but our preliminary results suggest that we have created a very different kind of film experience where students decompose, analyze, and assign meaning to visual events, and that these activities result in an increased ability to construct causal explanations of complex behavior.

\section{ACKNOWLEDGMENTS}

This work is funded by a Cognitive Studies in Educational Practice Grant from the James S. McDonnell Foundation, a Patricia Roberts Harris Fellowship, and a National Science Foundation training grant. Bill Sandoval and Iris Tabak are also 
core members of the BGuILE team. We would like to thank Pamela Lentine for assisting with the curriculum design and deployment, our teacher collaborators, Sharon Cannavino, Phil Hiller, Karen Olson, and Maria Vermiglio, and ecologists Hans Landel and David Scheel for their expertise on behavior and lions. Finally, we thank our anonymous reviewers for their helpful suggestions on improving this paper.

\section{REFERENCES}

[1] Anderson, J. R., Corbett, A. T., Koedinger, K. R., \& Pelletier, R. (1995). Cognitive tutors: Lessons learned. The Journal of the Learning Sciences, 4(2), 167-207.

[2] Baecker, R., Rosenthal, A. J., Friedlander, N., Smith, E., \& Cohen, A. (1996). A multimedia system for authoring motion pictures. In ACM Multimedia '96 Proceedings, (pp. 31-42). New York: ACM Press.

[3] Borovoy, R. D., Cooper, E. B. W., \& Bellamy, R. K. E. (1994). Media Fusion: An application of model-based communication. In Conference Companion to CHI '94, (pp. 1718). New York: ACM Press.

[4] Bransford, J. D., Franks, J. J., Vye, N. J., \& Sherwood, R. D. (1989). New approaches to instruction: Because wisdom can't be told. In S. Vosniadou \& A. Ortony (Eds.), Similarity and Analogical Reasoning (pp. 470-497). New York: Cambridge University Press.

[5] Brooks, K. M. (1996). Do story agents use rocking chairs? The theory and implementation of one model for computational narrative. In ACM Multimedia '96 Proceedings, (pp. 317-328). New York: ACM Press.

[6] Brown, E. \& Chignell, M. H. (1995). End user as developer: Free-form multimedia. In E. Barrett \& M. Redmond (Eds.), Contextual Media: Multimedia and Interpretation (pp. 189-211). Cambridge, MA: The MIT Press.

[7] Cappo, M. \& Darling, K. (1996). Measurement in Motion. Communications of the ACM, 39(8), 91-93.

[8] Erdman, B. (1990). The closely guided viewer: Form, style, and teaching in the educational film. In E. Ellsworth \& M. H. Whatley (Eds.), The Ideology of Images in Educational Media: Hidden Curriculums in the Classroom (pp. 27-42). New York: Teachers College Press.

[9] Fortner, R. W. (1985). Relative effectiveness of classroom and documentary film presentations on marine mammals. Journal of Research in Science Teaching, 21(2), 115-126.

[10] Friedler, Y., Zohar, A., \& Tamir, P. (1993). The effect of age and of learning on the ability to distinguish between anthropomorphic and teleological explanations. International Journal of Science Education, 15(4), 439-443.

[11] Gerba, D. (1984). A Study on Environmental Education Films. Unpublished undergraduate thesis, Syracuse University.

[12] Goldman-Segall, R. (1995). Deconstructing the HumptyDumpty myth: Putting it together to create cultural meaning. In E. Barrett \& M. Redmond (Eds.), Contextual Media: Multimedia and Interpretation (pp. 27-52). Cambridge, MA: The MIT Press.

[13] Koedinger, K. R., Anderson, J. R., Hadley, W. H., \& Mark, M. A. (1995). Intelligent tutoring goes to school in the big city. In J. Greer (Ed.), Proceedings of AI-ED 95: World Conference on Artificial Intelligence in Education, (pp. 421-428). Charlottesville, VA: Association for the Advancement of Computing in Education.
[14] Lima, S. L. \& Dill, L. M. (1990). Behavioral decisions made under the risk of predation: A review and prospectus. Canadian Journal of Zoology, 68, 619-640.

[15] Mackay, W. E. \& Davenport, G. (1989). Virtual video editing in interactive multimedia applications. Communications of the ACM, 32(7), 802-810.

[16] Nardi, B. A., Kuchinsky, A., Whittaker, S., Leichner, R., \& Schwarz, H. (1996). Video-as-data: Technical and social aspects of a collaborative multimedia application. Computer Supported Collaborative Work, 4, 73-100.

[17] NRC (1996). National Science Education Standards. Washington, DC: National Research Council.

[18] Pea, R. D. (1991). Learning through multimedia. IEEE Computer Graphics \& Applications, 11(4), 58-66.

[19] Pea, R. D. \& Gomez, L. M. (1992). Distributed multimedia learning environments: Why and how? Interactive Learning Environments, 2(2), 73-109.

[20] Rubin, A., Bresnahan, S., \& Ducas, T. (1996). Cartwheeling through CamMotion. Communications of the ACM, 39(8), 84-85.

[21] Rubin, A. \& Win, D. (1994). Studying motion with KidVid: A data collection and analysis tool for digitized video. In Conference Companion to CHI '94, (pp. 13-14). New York: ACM Press.

[22] Schaller, G. B. (1972). The Serengeti Lion: A Study of Predator-Prey Relations. Chicago, IL: University of Chicago Press.

[23] Schauble, L., Glaser, R., Duschl, R. A., Schulze, S., \& John, J. (1995). Students' understanding of the objectives and procedures of experimentation in the science classroom. The Journal of the Learning Sciences, 4(2), 131-166.

[24] Scheel, D. (1993). Waiting for lions in the grass: The usefulness of scanning and its effects during hunts. Animal Behaviour, 46, 695-704.

[25] Schofield, J. W. (1995). Computers and Classroom Culture. New York: Cambridge University Press.

[26] Schwab, R. G., Hart-Landsberg, S., Reder, S., \& Abel, M. (1992). Collaboration and constraint: Middle school teaching teams. In Proceedings of Computer Supported Collaborative Work 92, (pp. 241-248). New York: ACM Press.

[27] Silverstein, O. \& Tamir, P. (1993). The role of imagery in learning biology science through television. In Selected Readings from the Symposium of the International Visual Literacy Association, Delphi, Greece.

[28] Sinclair, A. R. E. (1995). Serengeti past and present. In A. R. E. Sinclair \& P. Arcese (Eds.), Serengeti II: Dynamics, Management, and Conservation of an Ecosystem (pp. 3-30). Chicago, IL: University of Chicago Press.

[29] Soula, J.-P., Baron, C., \& Nestor, C. (1997). A shift from chalk: Multimedia and instruction. IEEE Multimedia, 4(2), 5-9.

[30] Tabak, I., Smith, B. K., Sandoval, W. A., \& Reiser, B. J. (1996). Combining general and domain-specific strategic support for biological inquiry. In C. Frasson (Ed.), Proceedings of the Third International Conference on Intelligent Tutoring Systems, (pp. 288-296). London: Springer Verlag. 
[31] Tamir, P. \& Zohar, A. (1991). Anthropomorphism and teleology in reasoning about biological phenomena. Science Education, 75(1), 57-67.

[32] Wetzel, C. D., Radtke, P. H., \& Stern, H. W. (1994). Instructional Effectiveness of Video Media. Hillsdale, NJ: Lawrence Erlbaum Associates. 